\title{
SARS-Cov-2 pneumonia phenotyping on imaging exams of patients submitted to minimally invasive autopsy
}

\author{
Marcel Koenigkam-Santos ${ }^{1}$, Danilo Tadao Wada ${ }^{1}$, Maira Nilson Benatti ${ }^{2}$, Li Siyuan $^{3}$, \\ Sabrina Setembre Batah ${ }^{4}$, Andrea Antunes Cetlin², Marcelo Bezerra de Menezes ${ }^{2}$, \\ Alexandre Todorovic Fabro ${ }^{4}$
}

${ }^{1}$ Department of Medical Images, Hematology and Oncology, Ribeirao Preto Medical School, University of Sao Paulo, Campus Universitario Monte Alegre, Ribeirao Preto, SP, Brazil; ${ }^{2}$ Department of Internal Medicine, Ribeirao Preto Medical School, University of Sao Paulo, Campus Universitario Monte Alegre, Ribeirao Preto, SP, Brazil; ${ }^{3}$ Department of Surgery and Anatomy, Ribeirao Preto Medical School, University of Sao Paulo, Campus Universitario Monte Alegre, Ribeirao Preto, SP, Brazil; ${ }^{4}$ Department of Pathology and Legal Medicine, Ribeirao Preto Medical School, University of Sao Paulo, Campus Universitario Monte Alegre, Ribeirao Preto, SP, Brazil

Contributions: (I) Conception and design: M Koenigkam-Santos, AT Fabro; (II) Administrative support: M Koenigkam-Santos, de Menezes MB, AT Fabro; (III) Provision of study materials or patients: MN Benatti, AA Cetlin, de Menezes MB, AT Fabro; (IV) Collection and assembly of data: M Koenigkam-Santos, DT Wada, MN Benatti, L Siyuan, SS Batah; (V) Data analysis and interpretation: M Koenigkam-Santos, DT Wada, SS Batah, AT Fabro; (VI) Manuscript writing: All authors; (VII) Final approval of manuscript: All authors.

Correspondence to: Prof. Dr. Marcel Koenigkam-Santos. Department of Medical Images, Hematology and Oncology, Ribeirao Preto Medical School, University of Sao Paulo. Av. Bandeirantes 3900, Campus Universitario Monte Alegre, Ribeirao Preto 14048-900, SP, Brazil. Email: marcelk46@fmrp.usp.br; marcelk46@yahoo.com.br.

Background: Correlation between pathology and imaging of the new SARS-Cov-2 disease (COVID-19) is scarce. This study aimed to characterize SARS-Cov-2 pneumonia on imaging of patients submitted to minimally invasive autopsy (MIA).

Methods: This unicentric retrospective observational study included 46 consecutive patients with confirmed COVID-19 who underwent MIA. All clinical chest images were reviewed and classified for the presence and grade of viral pneumonia, as well as disease evolution. On CT, phenotypes were described as consistent with mild, moderate, or severe viral pneumonia, with or without radiological signs of organizing pneumonia (OP). In severe pneumonia, CT could also be classified as diffuse progressive OP or radiological diffuse alveolar damage (DAD). Specific features on CT were noted, including fibroproliferative signs that could indicate potential or initial fibrosis.

Results: MIA showed a heterogeneous panel of alterations, with a high prevalence of OP and acute fibrinous and organizing pneumonia (AFOP). Also, signs of interstitial fibrosis corresponded to the most prevalent pathological feature. Initial chest radiography (CXR) findings were mainly consistent with moderate or severe viral pneumonia. Most patients showed stability or improvement (reduction of opacities) on imaging. CTs were performed on 15 patients. Consolidations were found in most patients, frequently showing features consistent with an OP phenotype. Fibroproliferative changes were also prevalent on CT.

Conclusions: In this study, SARS-Cov-2 pneumonia showed heterogeneous radiological and pathological patterns. Signs of organization and potential or initial fibrosis were prevalent on both imaging and pathology. Imaging phenotyping may help to predict post-infection fibrosing interstitial pneumonitis in COVID-19.

Keywords: COVID-19; chest radiography (CXR); computed tomography; minimally invasive autopsy (MIA)

Submitted Aug 18, 2021. Accepted for publication Nov 26, 2021.

doi: $10.21037 /$ atm-21-4354

View this article at: https://dx.doi.org/10.21037/atm-21-4354

\footnotetext{
$\wedge$ ORCID: 0000-0002-7160-4691.
} 


\section{Introduction}

Imaging has played a crucial role in the evaluation of the new coronavirus SARS-Cov-2 disease (COVID-19), especially with chest radiography (CXR) and computed tomography (CT) for the diagnosis of viral pneumonia. Findings on CXR are considered difficult to interpret and typically described as bilateral and relatively symmetrical "patchy opacities", mostly peripheral and basal in distribution (1). As for most pulmonary diseases, CT is considered the gold standard imaging modality. Findings considered more characteristic of COVID-19 pneumonia are bilateral, peripheral, and basal predominant groundglass opacities (GGOs), with or without consolidations (2). Opacities may show a round morphology or be associated with the reversed halo sign, and crazy paving may also be present. Pleural effusions and lymphadenopathy occur in a small number of cases, may represent bacterial or fungal superinfection, and have been described as signs of a worse prognosis (3).

Several investigations have also described COVID-19 pneumonia evolution on imaging. In early-stage disease (04 days after symptoms onset), CT may show no pulmonary opacities or unspecific focal areas of GGO. At the middlestage of illness (5-13 days), CT has a higher sensitivity and usually shows the progression of lung opacities, with new or increasing consolidations and higher rates of bilateral multilobar involvement, also predominantly peripheral and basal. These findings peak around day ten after symptoms onset. Tomographic findings in late-stage (after 14 days) disease may be absent, but pulmonary opacities may stay up to several weeks and characterize a persistent pulmonary disease with fibrotic-like changes $(4,5)$.

Despite all studies describing imaging findings of SARS-Cov-2 pneumonia, pathologic analysis of patients infected with or who died of COVID-19 remains scarce. A good correlation of these findings with imaging features is occasionally reported. A recent literature review (6) described the utility of chest CT for prognostic evaluation of COVID-19, highlighting some imaging features that may indicate a pulmonary fibroproliferative process and predict persistent pulmonary abnormalities, but cited only a few studies based on clinical and radiological evaluation, without pathological correlation. Therefore, the exact role of imaging as a potential prediction tool of evolution from pneumonia to fibrosis is still unknown. In this study, we aimed to characterize the findings on imaging exams performed during clinical evaluation of patients who died with COVID-19 and were submitted to minimally invasive autopsy (MIA) in our institution, focusing on the adequate description of inflammation, organization, and potential/initial fibrosis on both methods. We present the following article in accordance with the STROBE reporting checklist (available at https://atm.amegroups.com/article/ view/10.21037/atm-21-4354/rc).

\section{Methods}

\section{Patients}

The study was conducted in accordance with the Declaration of Helsinki (as revised in 2013). The study was approved by the research ethics committee of the Ribeirao Preto Medical School University Hospital (CAAE.: 32475220.5.0000.5440). The requirement for informed consent was waived by the institutional review board as this study was part of a public health outbreak investigation. Clinical data were obtained from patients' electronic medical records.

This unicentric retrospective observational study included 46 consecutive patients with COVID-19 confirmed by real-time transcription polymerase chain reaction (RT-PCR) on oropharyngeal swab who underwent MIA. The autopsy was performed prospectively in consecutive patients recruited between April and September 2020, while the analysis of imaging exams was retrospective, using CXR and CT scans performed during routine clinical evaluation. All patients included in this study were referred to our high-complexity medical institution due to clinically moderate-severe disease for admission to a specialized ward or intensive care unit (ICU).

For all patients, the autopsy was done at the bedside, whiting 1 hour of death, and included obtaining several lung core fragments with the help of a matching 14-gauge cutting needle (Magnum Needles, Bard) and a biopsy gun (Magnum, Bard). Lung samples were randomly obtained from different spots and positions by the pathologists. Lung pathology specimens were fixed in formalin between 24 and 36 hours. Hematoxylin and eosin (H\&E)-stained sections were prepared, and histologic examination was performed with all available tissue obtained from the procedure. Histomorphological alterations were evaluated by specialized pulmonary pathologists, the most experienced one with ten years of practice in analyzing lung diseases. In all lung specimens, it was assigned the presence of hyaline membranes [the hallmark of diffuse alveolar damage (DAD)], plugs of organizing fibroblastic 
tissue [representing organizing pneumonia pattern (OP)], fibrin "balls" (indicating acute fibrinous and organizing pneumonia pattern (AFOP)], interstitial fibrotic changes, hemorrhagic infarct, and presence of arterial thrombosis. If more than one feature was present, the predominant ones were pointed out by the pathologists.

\section{Images' acquisition}

All exams included in this study were performed as part of the routine clinical evaluation. In our institution, as recommended, chest CT is not performed for all patients (7). Chest radiographs were performed in conventional equipment, mainly in the anteroposterior incidence.

Chest CT images were performed in multidetector scanners (Brilliance CT Big Bore 16 - Philips, Holland, or Aquilion Prime 160 - Toshiba, Japan), using similar protocols for the acquisition of high-resolution images of the lungs (8). Patients were scanned in the supine position. Volumetric axial images were obtained from the base to the apex of the lungs without the administration of intravenous contrast media. Typical acquisition parameters were: $120 \mathrm{kVp}$ tube voltage, $100-140$ ref mAs (auto current), 0.3-0.7 s gantry rotation time, reconstruction matrix size of $512 \times 512$, slice thickness, and increment of $1.0 \mathrm{~mm}$, using standard (soft) and hard kernel filters. Two patients included in this study performed angioCTs for the investigation of acute pulmonary thromboembolism. Acquisition parameters were similar, but exams were performed after intravenous bolus administration of 80 $100 \mathrm{~mL}$ of iodinated contrast media, using an automatic power injector, at a flow rate of $3-4 \mathrm{~mL} / \mathrm{s}$.

\section{Imaging evaluation}

Two thoracic radiologists, with 5 and 15 years of experience, independently reviewed all images in standard workstations, blinded to clinical data, laboratory, and pathology results. Divergences were later solved by consensus.

All CXRs available were reviewed and classified for the presence and grade of viral pneumonia, similarly as previously suggested (7): without pulmonary opacities; indeterminate for viral pneumonia; consistent with mild ("more black than white" lungs), moderate or severe ("more white than black") viral pneumonia. Pulmonary disease evolution on imaging was also evaluated, considering all exams from the initial to the last image before death, and classified as: radiological improvement (reduction or resolution of the pulmonary opacities and other alterations), stability, worsening (an increase in opacities), or probable secondary complication (significant changes on imaging pattern, like the appearance of new opacities mostly consistent with superimposed bacterial/fungal infection or pulmonary infarction, signs of barotrauma, pleural effusion).

Tomographic images were evaluated similarly to CXR images. First, the radiologists classified the exams as: without signs of viral pneumonia; indeterminate for viral pneumonia; or consistent with mild, moderate, or severe viral pneumonia. When consolidations were identified showing typical features such as predominant peribronchial or peripheral location, air bronchograms, a perilobular distribution, or relative subpleural sparing, the CT phenotype was classified as consistent with mild, moderate, or severe viral pneumonia with OP foci. Also, in cases of severe pneumonia, when appropriate, the CT pattern could be classified as diffuse progressive $\mathrm{OP}$ or radiological $\mathrm{DAD}$, this one typically showing pendent consolidations and the anteroposterior gradient. If the radiologist identified signs of complications on CT that could indicate bacterial/fungal infection or pulmonary infarct, such as lobar pneumonia, bronchopneumonia, inflammatory bronchiolitis, nodules, cavities, abscess, or empyema, this was also noted. At last, some specific features were annotated if present or not on CT images, focusing on the opacity's characteristics and signs of fibroproliferative alterations (indicating potential/ initial fibrosis) or complications: GGOs, consolidations, pendent consolidations, anteroposterior gradient, crazy paving, perilobular distribution, relative subpleural sparing, architectural distortion, traction bronchiectasis, pleural irregularity, honeycombing, pleural or pericardial effusion, and mediastinal lymphadenopathy.

\section{Statistical analysis}

Continuous variables are displayed as mean \pm standard deviation (SD) or median with interquartile ranges, and categorical variables are reported as counts and percentages. Comparisons between clinical, radiological, and pathological variables were made with Fischer exact test and Spearman rank correlation test. Data were analyzed with Graphpad Prism version 7.0 (GraphPad Software Inc., USA). P values $<0.05$ were considered statistically significant.

\section{Results}

The main clinical characteristics of the patients are 
Table 1 Main clinical characteristics of the 46 patients who performed minimally invasive autopsies and had chest imaging exams performed in our institution

\begin{tabular}{lc}
\hline Clinical characteristic & $\begin{array}{c}\text { Absolute (percentage) or } \\
\text { mean value (SD) }\end{array}$ \\
\hline Age (years) & $68( \pm 15)$ \\
Male:female & $23: 23$ \\
Comorbidity & $15(33 \%)$ \\
Diabetes & $25(54 \%)$ \\
Hypertension & $17(37 \%)$ \\
Obesity & $10(22 \%)$ \\
Cardiopathy & $15(33 \%)$ \\
Smoking exposition & $37(80 \%)$ \\
Intensive care unit admission & $19( \pm 11)$ \\
Days from symptoms onset to death & $13( \pm 10)$ \\
Days from RT-PCR+ to death & $14( \pm 10)$ \\
Days from ICU admission to death &
\end{tabular}

RT-PCR+, positive reverse transcriptase polymerase chain reaction; ICU, intensive care unit; SD, standard deviation.

presented in Table 1. There were as many men as women, and most patients had 60 or more years. Thirty-seven patients were admitted to an ICU at the beginning or during hospitalization. Hospitalization time varied considerably so that one patient died on the day of admission (early-stage disease), while others remained in the hospital for more than a month before death (late-stage disease). Cause of death as documented in electronic medical records varied, was commonly multiple and included: septic shock (62\%), acute kidney insufficiency (51\%), acute respiratory distress syndrome (ARDS) (48\%), myocardial infarction (26\%) and congestive heart failure (9\%). These causes were registered independently of the MIA results.

Table 2 summarizes general imaging evaluation and MIA findings for all 46 patients. Pathological analysis showed signs of reparation and organization related to OP or AFOP in most patients (81\%), while hyaline membranes indicating DAD was present in less than half of patients. Interstitial fibrosis was the most prevalent feature and was described mainly as the presence of loose fibroblastic proliferation associated with uniform inflammation of the alveolar wall (septal thickening), resembling a nonspecific interstitial pneumonia (NSIP) pattern. Different degrees of arterial thrombosis were described in half of the patients
Table 2 General imaging evaluation and minimally invasive autopsy results for all 46 patients included in this study

\begin{tabular}{lc}
\hline Initial CXR pattern & $\mathrm{N}[\%]$ \\
\hline No opacities & $3[7]$ \\
Indeterminate & $6[13]$ \\
Mild viral pneumonia & $3[7]$ \\
Moderate viral pneumonia & $17[37]$ \\
Severe viral pneumonia & $16[35]$ \\
Imaging evolution until death & \\
Improvement (fewer opacities) & $20[43]$ \\
Stability & $9[20]$ \\
Worsening (more opacities) & $7[15]$ \\
Complicated (changes in pattern and others) & $8[18]$ \\
Indeterminate & $2[4]$ \\
MIA features (presence of) & \\
Hyaline membranes & $21[46]$ \\
Plugs of organizing fibroblastic tissue & $20[44]$ \\
Fibrin "balls" & $32[70]$ \\
Interstitial fibrosis & $40[87]$ \\
Hemorrhagic infarct & $28[61]$ \\
Arterial thrombosis & $24[52]$ \\
\hline
\end{tabular}

CXR, chest radiography; MIA, minimally invasive autopsy.

and hemorrhagic infarct was also common, but not always present in the same patients. Imaging analysis showed that thirty-three patients (72\%) presented findings compatible with moderate or severe viral pneumonia on initial CXR. Despite the important pulmonary disease identified on initial CXR, most patients showed stability or improvement $(63 \%)$ on imaging during hospitalization before death. There was no significant correlation between initial CXR patterns and clinical or pathological data. Imaging improvement correlated with the presence of interstitial fibrosis $(\mathrm{P}=0.02)$ and arterial thrombosis $(\mathrm{P}<0.01)$ on MIA, while signs of complication on imaging additionally correlated with the presence of fibrin "balls" $(\mathrm{P}=0.04)$.

Results of the CT analysis are presented in Tables 3,4. Figures 1-3 show examples of the CT and MIA findings in three different patients. Acquisition of images was made in early or middle-stage disease for all 15 patients that performed a CT scan during hospitalization. The time between CT and death varied so that some patients 
Table 3 Chest computed tomography patterns (phenotypes) in correlation with the minimally invasive autopsy findings

\begin{tabular}{lcll}
\hline CT pattern (phenotype) & N cases & Main MIA features (N cases) \\
\hline Indeterminate for viral pneumonia & 3 & Hyaline membranes [2], plugs of organizing fibroblastic tissue [1] \\
Mild viral pneumonia & 2 & Hyaline membranes and fibrine "balls" [1], interstitial fibrosis [1] \\
Moderate viral pneumonia & 2 & $\begin{array}{l}\text { Plugs of organizing fibroblastic tissue and fibrine "balls" [1], } \\
\text { interstitial fibrosis [1] }\end{array}$ \\
Mild viral pneumonia with OP foci & 1 & Hemorrhagic infarct \\
Moderate viral pneumonia with OP foci & 2 & Fibrine "balls" [1] and interstitial fibrosis [1] \\
Severe viral pneumonia with OP foci & 2 & Hemorrhagic infarct [1], fibrine "balls" [1] \\
Severe viral pneumonia with DAD signs & 2 & All features including hemorrhagic infarct [1], hyaline membrane [1] \\
Severe viral pneumonia with diffuse progressive OP pattern & 1 & Interstitial fibrosis \\
\hline
\end{tabular}

CT, computed tomography; MIA, minimally invasive autopsy; OP, organizing pneumonia; DAD, diffuse alveolar damage.

Table 4 Imaging and minimally invasive autopsy features of the 15 patients who performed chest computed tomography exams during hospital stay in our institution

\begin{tabular}{|c|c|}
\hline CT features (presence of) & $\mathrm{N}[\%]$ \\
\hline Ground-glass opacities & 15 [100] \\
\hline Consolidations (any) & $13[87]$ \\
\hline Pendent consolidations & $6[40]$ \\
\hline Anteroposterior gradient & $2[13]$ \\
\hline Crazy paving & 5 [33] \\
\hline Perilobular distribution & $11[73]$ \\
\hline Relative subpleural sparing & $9[60]$ \\
\hline Architectural distortion & 8 [53] \\
\hline Traction bronchiectasis & $6[40]$ \\
\hline Pleural irregularity & 5 [33] \\
\hline Honeycombing & $1[7]$ \\
\hline Pleural effusion & $9[60]$ \\
\hline Pericardial effusion & 3 [20] \\
\hline Mediastinal lymphadenopathy & 5 [33] \\
\hline \multicolumn{2}{|l|}{ MIA features (presence of) } \\
\hline Hyaline membranes & $11[73]$ \\
\hline Plugs of organizing fibroblastic tissue & $7[47]$ \\
\hline Fibrin "balls" & $12[80]$ \\
\hline Interstitial fibrosis & 14 [93] \\
\hline Hemorrhagic infarct & 10 [67] \\
\hline Arterial thrombosis & $7[47]$ \\
\hline
\end{tabular}

CT, computed tomography; MIA, minimally invasive autopsy. had the exam done one or two days before death, while others died more than fifteen days after the exam (Table 3). In both patients who performed angioCTs, no signs of pulmonary embolism were found. Regarding imaging phenotyping on $\mathrm{CT}$, in three cases, the findings were considered indeterminate for viral pneumonia, all of them died during the middle-stage of illness, two presenting hyaline membranes as a main pathology finding, indicating DAD. Four cases were compatible with mild or moderate pneumonia on CT (without OP), only one showing hyaline membranes as a main pathological finding. Six patients were phenotyped with an OP component on CT. Four died during late-stage disease, two of them showing interstitial fibrosis as a main feature. Two patients showed severe pneumonia with DAD signs on CT, one died a day after (5 days after onset of symptoms) and showed an outspread of pathological inflammatory features including hyaline membranes, while the other died seven days after (during middle-stage disease) and showed hyaline membranes as a main finding. One patient was characterized with diffuse progressive OP pattern (severe pneumonia) on CT, died 18 days after the exam (late-stage disease), and showed important interstitial fibrosis (Figure 3). GGOs on CT were seen in all patients. Consolidations were found in most patients (13/15), frequently showing features consistent with OP foci (perilobular distribution or relative subpleural sparing) (Table 4). Features that may represent imminent/initial fibrosis (fibroproliferative alterations) on imaging were also prevalent (architectural distortion, traction bronchiectasis, and pleural irregularity), mainly architectural distortion that was identified in 8 exams. 

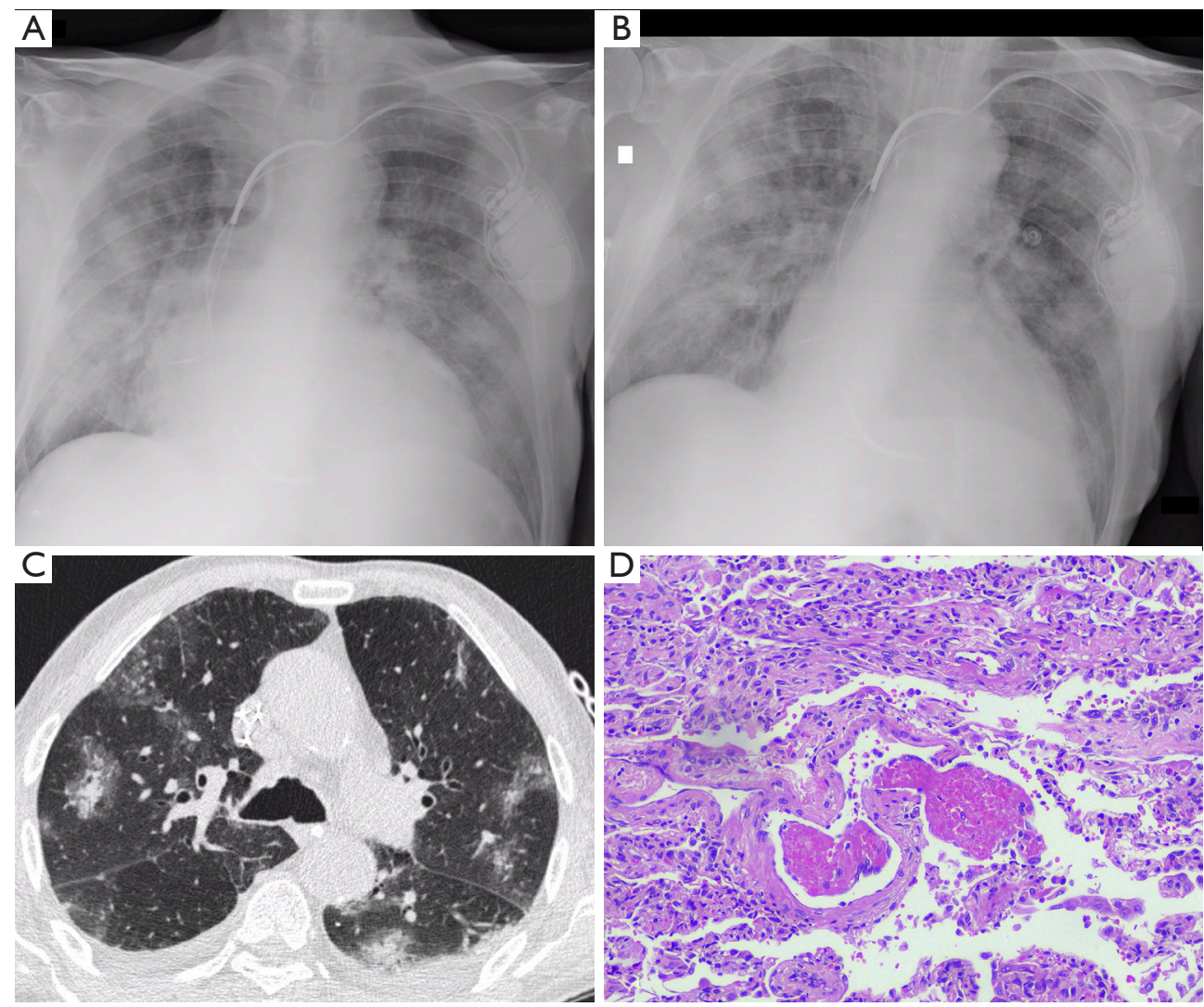

Figure 1 Example of a patient with COVID-19 pneumonia with features of organizing pneumonia. (A) and (B) show initial and last chest radiographies (CXR). Chest computed tomography (CT) [(C) axial image, lung window] was performed 2 days before minimally invasive autopsy (MIA) [(D) Hematoxylin and eosin-stained section at 20x magnification]. This patient had hypertension, diabetes and a cardiac pacemaker. Initial CXR was classified as showing moderate viral pneumonia (A) with improvement of imaging alterations before death, with less pulmonary opacities in the last CXR (B). CT was phenotyped as moderate viral pneumonia with organizing pneumonia foci (C). On CT images it was possible to identify multiple foci of consolidations, peribronchial and peripheral, with air bronchograms, perilobular distribution and relative subpleural sparing. There was also bilateral pleural effusion. MIA specimens showed interstitial fibrosis, and intraalveolar and intrabronchiolar fibrin aggregates in the form of fibrin "balls" (D) as major findings.

In only one case, honeycombing was detected, and both radiologists agreed that it could be related to an already present previous fibrotic alteration. Signs of interstitial fibrosis were described on MIA of $14 / 15$ patients who underwent CT exams. Significant correlations were observed between relative subpleural sparing and plugs of organizing fibroblastic tissue $(\mathrm{P}=0.04)$, as well as between architectural distortion and plugs of organizing fibroblastic tissue $(\mathrm{P}=0.04)$ and arterial thrombosis $(\mathrm{P}=0.04)$.

\section{Discussion}

In this study, CXR and CT images of 46 patients with COVID-19 submitted to MIA were evaluated. Pathology showed a heterogeneous panel of alterations, with a high prevalence of OP and AFOP. Signs of interstitial fibrosis corresponded to the most prevalent pathological feature. Most patients were referred to our institution for hospitalization showing moderate or severe pneumonia on initial CXR but evolved with stability or improvement of the opacities on imaging until death. This finding corroborates the idea that most patients in this study did not die directly because of the pulmonary disease but probably due to complications, possibly related to arterial thrombosis or hemorrhagic infarcts that were frequently found on MIA. Fifteen patients performed CT scans. In these cases, radiological and pathological findings were also heterogeneous, so that radiological DAD was not the most 

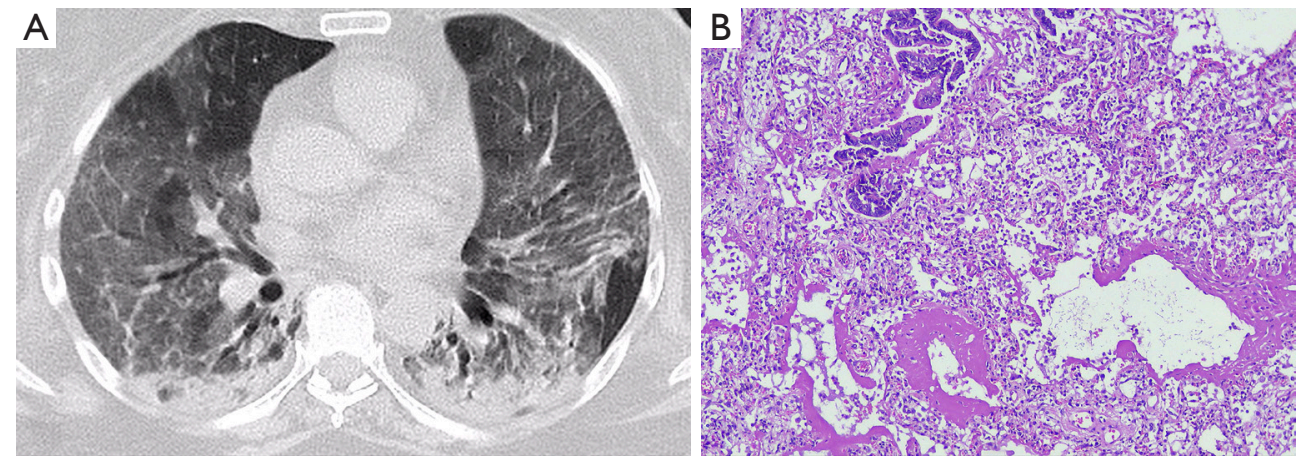

Figure 2 Chest computed tomography (CT) [(A) axial image, lung window] and microscopic evaluation [(B) Hematoxylin and eosinstained section at 10x magnification] of a patient with COVID-19 who deceased 1 day after performing the imaging exam. This patient had hypertension undergoing medical drug therapy. CT showed severe viral pneumonia with features of diffuse alveolar damage, including pendent consolidations and anteroposterior gradient (A). Minimally invasive autopsy specimens showed the presence of all inflammatory and fibrotic features, as well as hemorrhagic infarct and arterial thrombosis, with significative injury to the alveolar/capillary barrier characterizing hyaline membranes (B).
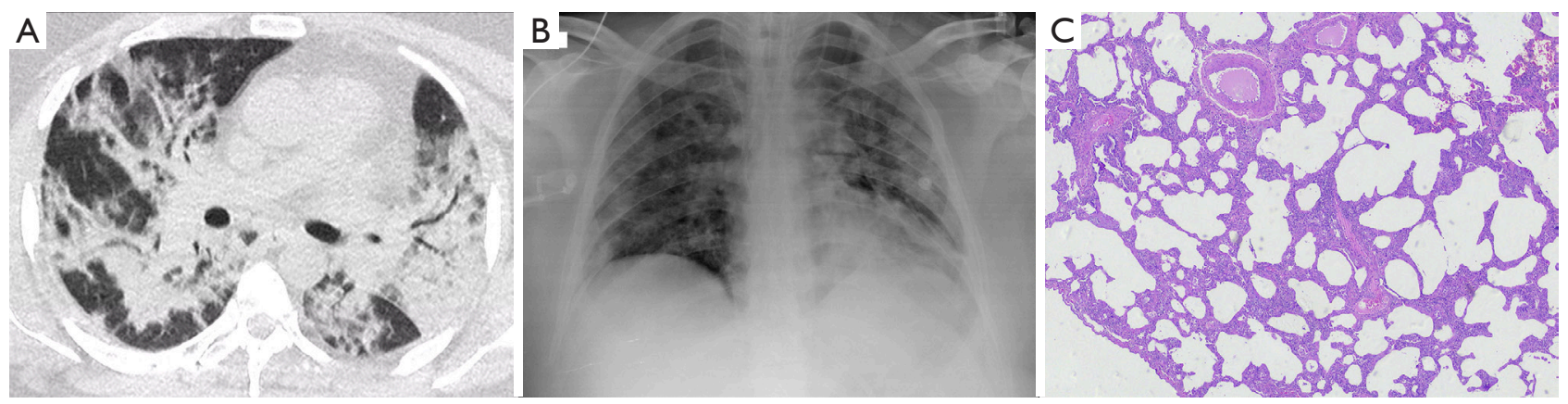

Figure 3 Chest computed tomography (CT) [(A) axial image, lung window], last chest radiography (B) and microscopic evaluation [(C) hematoxylin and eosin-stained section at 40x magnification] of a patient who died with COVID-19. This was an obese patient who died 18 days after performing the CT exam. CT images showed a severe viral pneumonia with a diffuse progressive organizing pneumonia pattern, presenting consolidations as the predominant opacity, central and peripheral, with perilobular distribution and relative subpleural sparing, as well as architectural distortion, traction bronchiectasis and pleural irregularity (A). In the evolution, there was improvement on imaging findings (fewer opacities) (B). Pathological specimens showed temporally homogeneous thickness of the alveolar septa by fibroblasts and collagen deposition as a main finding, in a non-specific interstitial pneumonia-like pattern (C).

prevalent finding. Radio-pathological signs of $\mathrm{OP}$ were prevalent, as well as fibroproliferative signs on imaging (architectural distortion, traction bronchiectasis, and pleural irregularity) and interstitial fibrosis on MIA. Despite the relatively small sample size, some significant correlations were observed.

Copin et al. (9) performed postmortem biopsies on six COVID-19 positive patients at different stages of the disease, in five of them, the predominant histologic pattern was AFOP. Ackermann et al. (10) examined seven lungs obtained during autopsy from patients who died from
COVID-19. The histologic pattern was DAD associated with severe endothelial injury and a widespread of thrombosis with microangiopathy in all cases. Wichmann et al. (11) performed postmortem CT and histopathologic analysis in 12 patients. Histomorphological DAD was seen in 8 patients, and a high incidence of thromboembolic events was also noted. Similar findings were described by McGonagle et al. (12) after autopsies of 10 African American decedents, with the cause of death attributed to COVID-19. Li et al. (13) studied 30 patients who died with COVID-19 using MIA and described that the most 
common manifestation was DAD in different stages. Flikweert et al. (14) performed postmortem needle core lung biopsies in 7 patients with COVID-19 related ARDS. Chest CT in all patients showed ground glass opacities, commonly progressing to nondependent consolidations, and four distinct histopathological patterns were described: AFOP, $\mathrm{OP}, \mathrm{DAD}$, and fibrosis. In our study, we have evaluated a larger series of patients when compared to previous studies, all of them with routine imaging exams to be analyzed, including 15 CT scans. Our results are more consistent with what Flikweert et al. (14) have described, highlighting COVID-19's heterogeneity and the importance of adequate characterization on imaging, identifying and describing the radiological signs of $\mathrm{OP}$ and potential or initial fibrosis $(15,16)$.

In COVID-19, radiological findings express the pulmonary damage caused by the SARS-CoV-2, initially represented by inflammation (viral pneumonia), that may evolve to a condition of acute lung injury (ALI), histologically represented by reparation and organization in the form of DAD, OP or AFOP $(17,18)$. Moreover, a thrombotic injury mediated by activation of complement pathways has also been observed $(19,20)$. Organization, characterized by fibroblast proliferation, has long been recognized as an important pattern of response in ALI, which may lead to repair and return to normal lung architecture or evolve to fibrosis $(21,22)$. At imaging, OP is mainly represented by bilateral consolidations, with air bronchograms, associated or not with GGO and nodules, predominantly peripheral or peribronchial, typically with a perilobular distribution or relative subpleural sparing (23). Acute fibrinous and organizing pneumonia is considered a rare form of ALI and is characterized in histology by the dominant presence of the "fibrin balls" within the alveolar spaces. It is a pattern more recently described, with fewer studies available in the literature, especially concerning its radiological aspects (24). In this study, radiological and pathological findings were described in agreement with previous knowledge regarding these inflammatory patterns in the lungs. Different from most previous studies, DAD (radiological and pathological) was not the only pattern identified in our patients. On the other hand, consolidations showing radiological features of $\mathrm{OP}$ and pathological $\mathrm{OP} / \mathrm{AFOP}$ were prevalent, as well as tomographic fibroproliferative alterations and pathological signs of interstitial fibrosis. Arterial thrombosis on MIA correlated with the presence of imaging improvement (reduction of pulmonary opacities) and architectural distortion on
CT, but a more precise correlation between the presence of thrombosis and hemorrhagic infarction and imaging findings could not be obtained in this study.

Several studies have shown that COVID-19-associatedARDS is a heterogeneous disorder, and pulmonary fibrosis may develop, as well as previously described for other viral infections (25). Some authors suggest that the "classical" DAD pattern is more related to high pulmonary elastance (low compliance), high weight, and high recruitability, while a pattern with a diffuse progressive OP pattern is probably related to a low pulmonary elastance (high compliance), low weight and low recruitability. Therefore, this distinction is important and may have immediate therapeutic repercussions, and imaging may help $(26,27)$. There are also other studies showing that adequate characterization of the opacities and a pattern categorization on CT can be important, highlighting the presence and extent of consolidations as a potential prognostic feature in the imaging evaluation of the disease (28-30). At last, a very recent document (31) from the European Society of Thoracic Imaging (ESTI) and the European Society of Radiology (ESR) discusses the role of imaging in the longterm follow-up of COVID-19 patients. In this document, authors describe that post-COVID-19 pneumonia changes are mainly consistent with prior organizing pneumonia and are likely to disappear within 12 months of recovery from the acute infection in most patients. This also highlights the importance of identifying and describing the features of $\mathrm{OP}$ in the acute phase imaging evaluation, as presented and discussed in this work.

This study has some limitations. First, it was a retrospective analysis in a single center with a small sample size, which may not reflect the overall clinical findings. The decision to perform imaging exams was based on clinical judgment as part of the routine evaluation of the patients, which may create bias on imaging evaluation and correlation. Also, only 15 patients had CT images to be analyzed, and the time between CT and death varied greatly. Ideally, for a more precise correlation, more CT scans performed closely to death would be necessary. MIA was performed by the pathologists, but blindly, without the help of any imaging exam to guide, which could also have improved the quality of the tissue sampling.

In conclusion, what still needs to be adequately understood is why and how, in COVID-19 pneumonia, some patients progress from viral pneumonia to organization and eventually to pulmonary fibrosis, with different presentations. The role of imaging as a potential 
prediction tool of evolution to fibrosis is yet to be established, but this study helps to strengthen the concept that adequately categorization (phenotyping) of viral pneumonia and its progression is important, that it may help to predict which patients will develop post-infection fibrosing interstitial pneumonitis.

\section{Acknowledgments}

Funding: None.

\section{Footnote}

Reporting Checklist: The authors have completed the STROBE reporting checklist. Available at https://atm. amegroups.com/article/view/10.21037/atm-21-4354/rc

Data Sharing Statement: Available at https://atm.amegroups. com/article/view/10.21037/atm-21-4354/dss

Conflicts of Interest: All authors have completed the ICMJE uniform disclosure form (available at https://atm. amegroups.com/article/view/10.21037/atm-21-4354/coif). The authors have no conflicts of interest to declare.

Ethical Statement: The authors are accountable for all aspects of the work in ensuring that questions related to the accuracy or integrity of any part of the work are appropriately investigated and resolved. The study was conducted in accordance with the Declaration of Helsinki (as revised in 2013). The study was approved by the research ethics committee of the Ribeirao Preto Medical School University Hospital (CAAE.: 32475220.5.0000.5440). The requirement for informed consent was waived by the institutional review board as this study was part of a public health outbreak investigation.

Open Access Statement: This is an Open Access article distributed in accordance with the Creative Commons Attribution-NonCommercial-NoDerivs 4.0 International License (CC BY-NC-ND 4.0), which permits the noncommercial replication and distribution of the article with the strict proviso that no changes or edits are made and the original work is properly cited (including links to both the formal publication through the relevant DOI and the license). See: https://creativecommons.org/licenses/by-ncnd/4.0/.

\section{References}

1. Chang D, Lin M, Wei L, et al. Epidemiologic and Clinical Characteristics of Novel Coronavirus Infections Involving 13 Patients Outside Wuhan, China. JAMA 2020;323:1092-3.

2. Bernheim A, Mei X, Huang $M$, et al. Chest CT Findings in Coronavirus Disease-19 (COVID-19): Relationship to Duration of Infection. Radiology 2020;295:200463.

3. Shi H, Han X, Jiang N, et al. Radiological findings from 81 patients with COVID-19 pneumonia in Wuhan, China: a descriptive study. Lancet Infect Dis 2020;20:425-34.

4. Pan F, Ye T, Sun P, et al. Time Course of Lung Changes at Chest CT during Recovery from Coronavirus Disease 2019 (COVID-19). Radiology 2020;295:715-21.

5. Chung M, Bernheim A, Mei X, et al. CT Imaging Features of 2019 Novel Coronavirus (2019-nCoV). Radiology 2020;295:202-7.

6. Machnicki S, Patel D, Singh A, et al. The Usefulness of Chest CT Imaging in Patients With Suspected or Diagnosed COVID-19: A Review of Literature. Chest 2021;160:652-70.

7. Revel MP, Parkar AP, Prosch H, et al. COVID-19 patients and the radiology department - advice from the European Society of Radiology (ESR) and the European Society of Thoracic Imaging (ESTI). Eur Radiol 2020;30:4903-9.

8. Raghu G, Remy-Jardin M, Myers JL, et al. Diagnosis of Idiopathic Pulmonary Fibrosis. An Official ATS/ERS/JRS/ ALAT Clinical Practice Guideline. Am J Respir Crit Care Med 2018;198:e44-68.

9. Copin MC, Parmentier E, Duburcq T, et al. Time to consider histologic pattern of lung injury to treat critically ill patients with COVID-19 infection. Intensive Care Med 2020;46:1124-6.

10. Ackermann M, Verleden SE, Kuehnel M, et al. Pulmonary Vascular Endothelialitis, Thrombosis, and Angiogenesis in Covid-19. N Engl J Med 2020;383:120-8.

11. Wichmann D, Sperhake JP, Lütgehetmann M, et al. Autopsy Findings and Venous Thromboembolism in Patients With COVID-19: A Prospective Cohort Study. Ann Intern Med 2020;173:268-77.

12. McGonagle D, Plein S, O'Donnell JS, et al. Increased cardiovascular mortality in African Americans with COVID-19. Lancet Respir Med 2020;8:649-51.

13. Li Y, Wu J, Wang S, et al. Progression to fibrosing diffuse alveolar damage in a series of 30 minimally invasive autopsies with COVID-19 pneumonia in Wuhan, China. Histopathology 2021;78:542-55. 
14. Flikweert AW, Grootenboers MJJH, Yick DCY, et al. Late histopathologic characteristics of critically ill COVID-19 patients: Different phenotypes without evidence of invasive aspergillosis, a case series. J Crit Care 2020;59:149-55.

15. Muller N. Pulmonary infections in diseases of the lung. In: Muller N, editor. Muller's Diseases of the Lung: Radiologic and Pathologic Correlations: Lippincott Williams \&Wilkins ed; 2003. p. 17-75.

16. Franquet T. Imaging of pulmonary viral pneumonia. Radiology 2011;260:18-39.

17. Zhang H, Zhou P, Wei Y, et al. Histopathologic Changes and SARS-CoV-2 Immunostaining in the Lung of a Patient With COVID-19. Ann Intern Med 2020;172:629-32.

18. Xu Z, Shi L, Wang Y, et al. Pathological findings of COVID-19 associated with acute respiratory distress syndrome. Lancet Respir Med 2020;8:420-2.

19. Varga Z, Flammer AJ, Steiger P, et al. Endothelial cell infection and endotheliitis in COVID-19. Lancet 2020;395:1417-8.

20. Kim EA, Lee KS, Primack SL, et al. Viral pneumonias in adults: radiologic and pathologic findings. Radiographics 2002;22 Spec No:S137-49.

21. Strieter RM, Mehrad B. New mechanisms of pulmonary fibrosis. Chest 2009;136:1364-70.

22. Kligerman SJ, Franks TJ, Galvin JR. From the radiologic pathology archives: organization and fibrosis as a response to lung injury in diffuse alveolar damage, organizing pneumonia, and acute fibrinous and organizing pneumonia. Radiographics 2013;33:1951-75.

23. Drakopanagiotakis F, Paschalaki K, Abu-Hijleh M, et al.

Cite this article as: Koenigkam-Santos M, Wada DT, Benatti MN, Siyuan L, Batah SS, Cetlin AA, de Menezes MB, Fabro AT. SARS-Cov-2 pneumonia phenotyping on imaging exams of patients submitted to minimally invasive autopsy. Ann Transl Med 2022;10(3):140. doi: 10.21037/atm-21-4354
Cryptogenic and secondary organizing pneumonia: clinical presentation, radiographic findings, treatment response, and prognosis. Chest 2011;139:893-900.

24. Tian S, Xiong Y, Liu H, et al. Pathological study of the 2019 novel coronavirus disease (COVID-19) through postmortem core biopsies. Mod Pathol 2020;33:1007-14.

25. Spagnolo P, Balestro E, Aliberti S, et al. Pulmonary fibrosis secondary to COVID-19: a call to arms? Lancet Respir Med 2020;8:750-2.

26. Gattinoni L, Chiumello D, Caironi P, et al. COVID-19 pneumonia: different respiratory treatments for different phenotypes? Intensive Care Med 2020;46:1099-102.

27. Rello J, Storti E, Belliato M, et al. Clinical phenotypes of SARS-CoV-2: implications for clinicians and researchers. Eur Respir J 2020;5 5:2001028.

28. Tabatabaei SMH, Talari H, Moghaddas F, et al. CT Features and Short-term Prognosis of COVID-19 Pneumonia: A Single-Center Study from Kashan, Iran. Radiol Cardiothorac Imaging 2020;2:e200130.

29. Li Y, Shang K, Bian W, et al. Prediction of disease progression in patients with COVID-19 by artificial intelligence assisted lesion quantification. Sci Rep 2020;10:22083.

30. Jin C, Tian C, Wang Y, et al. A Pattern Categorization of CT Findings to Predict Outcome of COVID-19 Pneumonia. Front Public Health 2020;8:567672.

31. Martini K, Larici AR, Revel MP, et al. COVID-19 pneumonia imaging follow-up: when and how? A proposition from ESTI and ESR. Eur Radiol 2021. [Epub ahead of print]. 